\title{
A Novel Method for HBT Intrinsic Collector Resistance Extraction from S-parameters
}

\author{
Johansen, Tom Keinicke; Krozer, Viktor; Hadziabdic, Dzenan; Jiang, Chenhui; Konczykowska, \\ Agnieszka; Dupuy, Jean-Yves
}

Published in:

Asia-Pacific Microwave Conference, 2007. APMC 2007.

Link to article, DOI:

10.1109/APMC.2007.4555014

Publication date:

2007

Document Version

Publisher's PDF, also known as Version of record

Link back to DTU Orbit

Citation $(A P A)$ :

Johansen, T. K., Krozer, V., Hadziabdic, D., Jiang, C., Konczykowska, A., \& Dupuy, J-Y. (2007). A Novel Method for HBT Intrinsic Collector Resistance Extraction from S-parameters. In Asia-Pacific Microwave Conference, 2007. APMC 2007. (Vol. 1-5, pp. 1927-1930). IEEE. https://doi.org/10.1109/APMC.2007.4555014

\section{General rights}

Copyright and moral rights for the publications made accessible in the public portal are retained by the authors and/or other copyright owners and it is a condition of accessing publications that users recognise and abide by the legal requirements associated with these rights.

- Users may download and print one copy of any publication from the public portal for the purpose of private study or research.

- You may not further distribute the material or use it for any profit-making activity or commercial gain

- You may freely distribute the URL identifying the publication in the public portal 


\title{
A Novel Method for HBT Intrinsic Collector Resistance Extraction from S-Parameters
}

\author{
Tom Johansen*, Viktor Krozer*, Dzenan Hadziabdic*, Chenhui Jiang*, Agnieszka Konczykowska ${ }^{\dagger}$, Jean-Yves Dupuy ${ }^{\dagger}$ \\ ${ }^{*}$ Oersted-DTU, Electro-Science Section Systems \\ Technical University of Denmark \\ 2800 Kgs. Lyngby, Denmark \\ Email: tkj@oersted.dtu.dk \\ †Alcatel-Thales III-V Lab \\ F-91461 Marcoussis Cedex, France
}

\begin{abstract}
In this paper the extraction of series resistances for high-speed InP DHBT devices is investigated. Known extraction methods based on measured S-parameters are reviewed and error terms are identified. A novel method for intrinsic collector resistance extraction is proposed. The method is based on $\mathbf{S}$ parameters measured in saturation and forward active regions. The results are applied to the large-signal modeling of InP DHBT devices using the UCSD HBT model and very accurate model response is obtained.
\end{abstract}

\section{INTRODUCTION}

InP based double heterojunction bipolar transistors (DHBTs) has long been recognized for its high potential in ultrahigh-speed microelectronic applications. For accurate circuit simulation several InP DHBT specific modeling issues must be taken into account. First, the forward transit time and base-collector capacitance experience modulation with bias even in the low current regime. Secondly, the base-collector heterojunction behavior may influence the characteristics in the saturation region due to the current blocking effect. Largesignal models such as VBIC95, HICUM, and MEXTRAM, developed for Si-based bipolar devices, can in general not accurately model the additional mechanisms observed in InP DHBT devices. The UCSD HBT model was developed for III-V devices to take the bias modulation of the forward transit time and base-collector capacitance into account [1]. The model is, however, still limited in its accuracy in the saturation region for InP DHBT due to the current blocking effect.

Traditionally the total collector resistance, being the cumulative resistance of semiconductor layers from the contact to intrinsic base-collector junction, has been extracted from the static $I_{c}-V_{c e}$ characteristic in the saturation region [2]. The presence of the current blocking effect in InP DHBTs, however, can often be mistaken for a too large intrinsic collector resistance. An alternative method of intrinsic collector resistance extraction avoiding the current barrier effect would clearly be beneficial. In [3] a method based on Sparameter measurements in the cut-off region is proposed as a way to extract the intrinsic collector resistance. In the cut-off region, however, the relative low intrinsic collector



Fig. 1. Small-signal equivalent circuit model.

resistance is seen in series with a high-impedance due to the intrinsic base-collector capacitance making the extraction based on experimental data difficult. Instead we have observed a strong dependence of the real part of the $Z_{12}$-parameter in saturation on the intrinsic collector resistance. The presence of an intrinsic collector resistance leads to an overestimation of the emitter resistance obtained with the well-known Sparameters method [4]. Instead the emitter-resistance should be extracted from the real part of the $Z_{12}$-parameter in the forward active region. Applying an open-collector condition for the saturation region measurements prevents the current blocking effect from occurring allowing accurate extraction of the 'true' intrinsic collector resistance once the emitter resistance is known.

In this paper accurate extraction methods for the series resistances needed for the large-signal modeling of InP DHBT devices is investigated. Particular emphasis will be given on the extraction of the intrinsic collector resistance from S-parameters. The extraction is performed on an integrated InP/InGaAs DHBT device with emitter area $10 \times 1.5 \mu \mathrm{m}^{2}$ fabricated at the Alcatel-Thales III-V laboratory. The device exhibits $200 / 210 \mathrm{GHz} f_{t} / f_{\max }$ and breakdown voltage $\mathrm{BV}$ ceo $>7 \mathrm{~V}$. 


\section{ExtRaction METHOD}

A hybrid- $\Pi$ small-signal equivalent circuit model for the integrated InP DHBT devices is shown in Fig. 1. The dynamic junction resistances $R_{b c}$, and $R_{b c x}$ are included in the model to be able to describe the small-signal behavior even in the saturation region. Neglecting the influence of the collectoremitter overlap capacitance $C_{c e o}$ the analytical expressions for the Z-parameters are

$$
\begin{aligned}
Z_{11}= & \frac{Z_{b e}}{1+g_{m} Z_{b e}}+\frac{R_{b i} Z_{b c}}{\left(R_{b i}+R_{c i}+Z_{b c}+Z_{b c x}\right)\left(1+g_{m} Z_{b e}\right)}+ \\
& \frac{R_{b i}\left(R_{c i}+Z_{b c x}\right)}{R_{b i}+R_{c i}+Z_{b c}+Z_{b c x}}+R_{b x}+R_{e} \\
Z_{12}= & \frac{Z_{b e}}{1+g_{m} Z_{b e}}+\frac{R_{b i} Z_{b c}}{\left(R_{b i}+R_{c i}+Z_{b c}+Z_{b c x}\right)\left(1+g_{m} Z_{b e}\right)}+ \\
& \frac{R_{b i} R_{c i}}{R_{b i}+R_{c i}+Z_{b c}+Z_{b c x}}+R_{e} \\
Z_{21}= & \frac{Z_{b e}}{1+g_{m} Z_{b e}}+\frac{R_{b i} Z_{b c}-g_{m} Z_{b c} Z_{b c x} Z_{b e}}{\left(R_{b i}+R_{c i}+Z_{b c}+Z_{b c x}\right)\left(1+g_{m} Z_{b e}\right)}+ \\
& \frac{R_{b i} R_{c i}}{R_{b i}+R_{c i}+Z_{b c}+Z_{b c x}}+R_{e} \\
Z_{22}= & \frac{Z_{b e}}{1+g_{m} Z_{b e}}+\frac{Z_{b c}\left(R_{b i}+Z_{b c x}\right)}{\left(R_{b i}+R_{c i}+Z_{b c}+Z_{b c x}\right)\left(1+g_{m} Z_{b e}\right)}+ \\
& \frac{R_{c i}\left(R_{b i}+Z_{b c x}\right)}{R_{b i}+R_{c i}+Z_{b c}+Z_{b c x}}+R_{c x}+R_{e}
\end{aligned}
$$

where $Z_{b e}=R_{b e}\left\|\frac{1}{j \omega C_{b e}}, Z_{b c}=R_{b c}\right\| \frac{1}{j \omega C_{b c}}$, and $Z_{b c x}=$ $R_{b c x} \| \frac{1}{j \omega C_{b o x}}$ have been used for compact notation.

\section{A. Saturation region extraction}

Gobert et al. [4] reported a method of extracting the access resistances $R_{b x}, R_{e}$, and $R_{c x}$ for $\mathrm{HBT}$ devices by linear extrapolation versus inverse base current $1 / I_{b}$ of the real parts of $Z_{11}-Z_{12}, Z_{12}, Z_{22}-Z_{12}$, respectively, determined from saturation region $S$-parameters. Extrapolation to infinity base currents forces the dynamic resistances of all junctions towards zero or equivalent $Z_{b e} \rightarrow 0, Z_{b c} \rightarrow 0$, and $Z_{b c x} \rightarrow 0$. Under these conditions the analytical Z-parameter expressions shows that

$$
\begin{aligned}
& \operatorname{Re}\left(Z_{11}-Z_{12}\right)=R_{b x} \\
& \operatorname{Re}\left(Z_{12}\right)=\frac{R_{c i} R_{b i}}{R_{c i}+R_{b i}}+R_{e} \\
& \operatorname{Re}\left(Z_{22}-Z_{12}\right)=R_{c x}
\end{aligned}
$$

The presence of an intrinsic collector is observed to give rise to an overestimation of the emitter resistance. The extrapolated value depends on the parallel combination of the intrinsic base resistance $R_{b i}$ and intrinsic collector resistance $R_{c i}$ and do not represent the emitter access resistance.

In Fig. 2 the evolution of $\operatorname{Re}\left(Z_{11}-Z_{12}\right), \operatorname{Re}\left(Z_{12}\right)$, and $\operatorname{Re}\left(Z_{22}-Z_{12}\right)$ versus inverse base current $1 / I_{b}$ for our InP

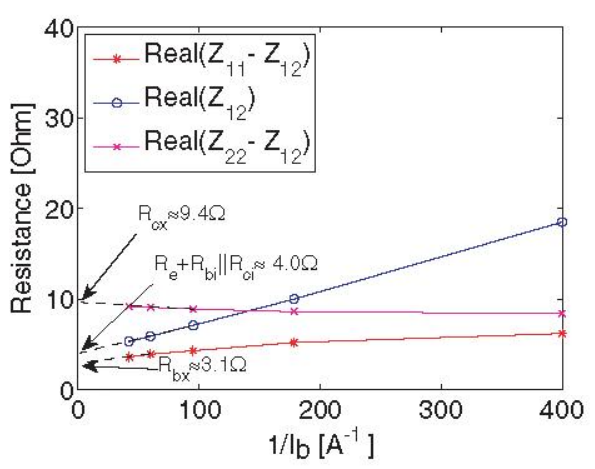

Fig. 2. Evolution of $\operatorname{Re}\left(Z_{11}-Z_{12}\right), \operatorname{Re}\left(Z_{12}\right)$, and $\operatorname{Re}\left(Z_{22}-Z_{12}\right)$ versus inverse base current $1 / I_{b}\left(I_{c}=0\right)$.

DHBT device is shown. The extraction is performed on deembedded S-parameters measured in the saturation region with the collector current forced to zero $\left(I_{c}=0\right)$.

\section{B. Forward active region extraction}

Dvorak et al. [5] described an alternative extraction method for $R_{e}$ based on linear extrapolation versus inverse emitter current $1 / I_{e}$ of the real part of $Z_{12}$ measured in the forward active region. At very large collector currents $I_{c} \rightarrow \infty$ the dynamic resistance of the base-emitter junction is forced towards zero. The intrinsic and extrinsic base-collector junctions, however, will remain reversed biased and the dynamic resistances can be assumed to be infinity. In this case we have $Z_{b c}=\frac{1}{j \omega C_{b c}}$, and $Z_{b c x}=\frac{1}{j \omega C_{b o x}}$. Under these conditions the analytical Zparameter expressions evaluated at low frequencies shows that

$$
\operatorname{Re}\left(Z_{12}(\omega \approx 0)\right)=\frac{R_{b i} C_{b c x}}{\left(C_{b c x}+C_{b c}\right)(1+\beta)}+R_{e}
$$

where the relation $g_{m} R_{b e}=\beta$ has been used. Again it is observed that the extrapolated value overestimates the emitter resistance. This is especially true for InP DHBT devices with typical $\beta$ values around $20-30$. The extraction of the emitter resistance can be improved by calculating the first term in eq. (8) and subtracting it from $\operatorname{Re}\left(Z_{12}\right)$. Because of the bias modulation of the intrinsic base-collector capacitance, it is necessary to calculate this term at all bias points used for the extrapolation. The extracted intrinsic and extrinsic basecollector capacitances for the InP DHBT device considered in this work is shown Fig. 3. The extraction is based on Z-parameters de-embedded for base and collector access resistances ( $R_{b x}$, and $R_{c x}$ ) using the method described in [6]. Because the extraction of the base-collector capacitance in [6] depends only on differences between the Z-parameters there is no influence from the yet unknown emitter resistance. The intrinsic base resistance $R_{b i}$ is evaluated from the improved semi-impedance circle method [7]. The extracted intrinsic base resistance versus frequency of our InP DHBT device is shown in Fig. 4 at the bias point $\left(I_{c}=27.9 \mathrm{~mA}, V_{c e}=1.5 \mathrm{~V}\right)$. As the intrinsic base resistance is fairly independent of bias for InP DHBT devices the value at the shown bias point is used for 


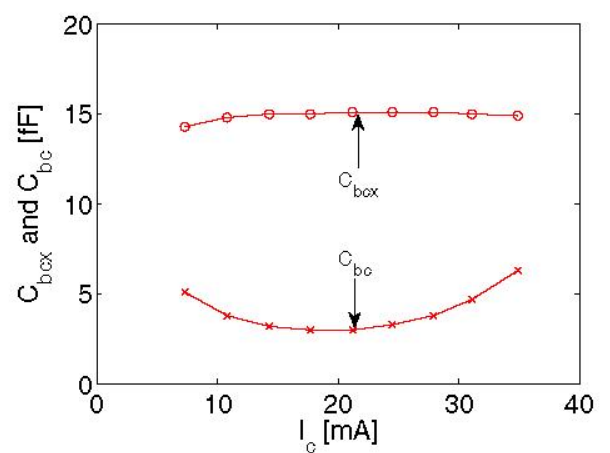

Fig. 3. Intrinsic and extrinsic base-collector capacitance versus collector current $\left(V_{c e}=1.5 \mathrm{~V}\right)$.

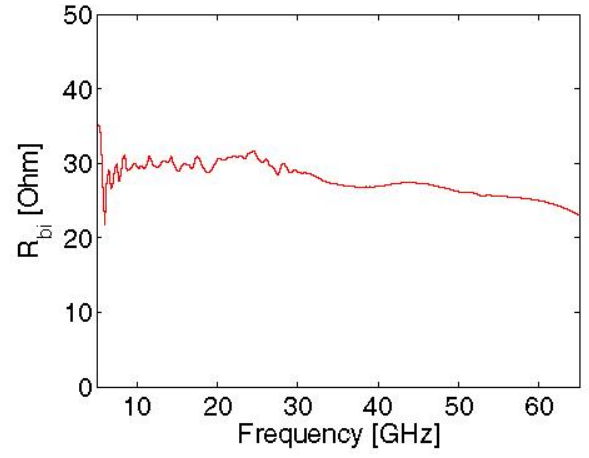

Fig. 4. Intrinsic base resistance versus frequency $\left(I_{c}=27.9 \mathrm{~mA}, V_{c e}=\right.$ $1.5 \mathrm{~V})$.

the correction. The value of $\beta$ is estimated from the value of $g_{m} R_{b e}$ extracted at low frequency.

The evolution of $\operatorname{Re}\left(Z_{12}\right)$ versus inverse collector current for the InP DHBT device is shown in Fig. 5. Before correction the extrapolated value of approximately $2.5 \Omega$ is obtained. After correction a value for the emitter access resistance of $R_{e} \approx 1.8 \Omega$ is determined. This value is significantly lower than the extrapolated value found from the saturation region measurements $(\approx 4.0 \Omega)$ clearly demonstrating the influence from the intrinsic collector resistance on the emitter access resistance determination.

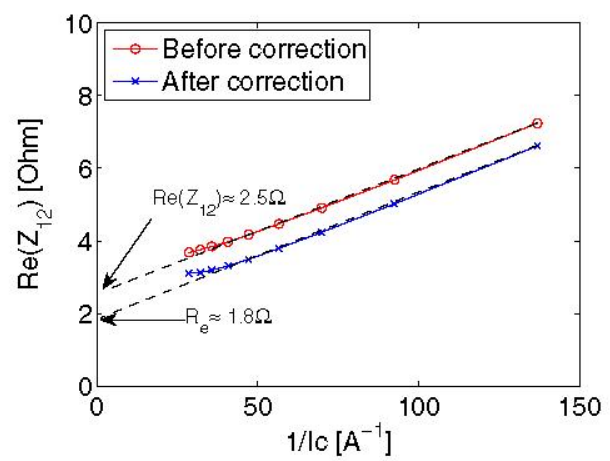

Fig. 5. Evolution of $\operatorname{Re}\left(Z_{12}\right)$ versus inverse collector current $1 /$ Ic $\left(V_{c e}=\right.$ $1.5 \mathrm{~V})$.

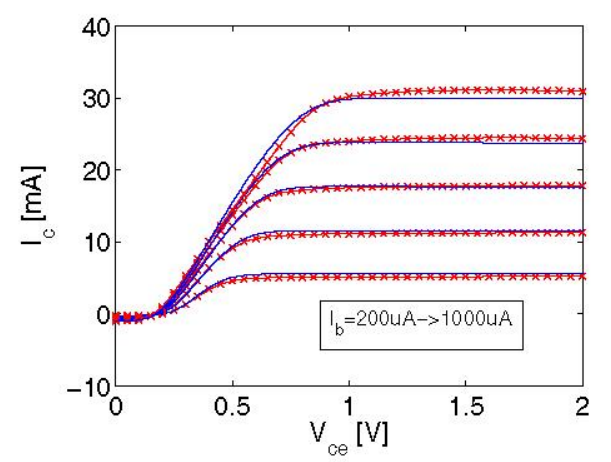

Fig. 6. $I_{C}-V_{c e}$ characteristics. Symbols: measurements, solid line: model response.

\section{Intrinsic collector resistance extraction}

After the access emitter resistance $R_{e}$ has been found from the corrected extrapolation of $\operatorname{Re}\left(Z_{12}\right)$ using forward active region data the intrinsic collector resistance can be determined from the corresponding extrapolated value, called $\operatorname{Re}\left(Z_{12}^{s a t}\right)$, in the saturation region as

$$
R_{c i}=\frac{\left(\operatorname{Re}\left(Z_{12}^{s a t}\right)-R_{e}\right) R_{b i}}{R_{b i}-\left(\operatorname{Re}\left(Z_{12}^{s a t}\right)-R_{e}\right)}
$$

Inserting previously determined values gives $R_{c i} \approx 2.4 \Omega$. This is the intrinsic collector resistance at forward biased basecollector junction where the depletion region is narrow. The current blocking effect should not influence the result because the open-collector biasing condition is used.

\section{LARGE-Signal Modeling REsults}

The extracted series resistances have been included into the UCSD HBT large-signal model. The model parameters has been extracted over temperature and self-heating effects is taken into account. As a verification of the intrinsic collector resistance extraction the $I_{c}-V_{c e}$ characteristics is shown in Fig.6. The correct slope at lower current levels in the saturation region is obtained without any further optimization of the intrinsic collector resistance. At higher current level a small difference in slope is observed and this is due to the collector blocking effect.

Fig. 7 and Fig. 8 shows the cutoff frequency and total basecollector capacitance versus collector current, respectively. The transit-time parameters of the UCSD HBT model are extracted with emphasis on the simultaneous fitting of the cutoff frequency and base-collector capacitance versus collector current. A compact representation of the S-parameters versus frequency for the bias point $I_{c}=24.5 \mathrm{~mA}, V_{c e}=1.5 \mathrm{~V}$ close to peak- $f_{T}$ for the InP DHBT device is shown in Fig. 9. The excellent agreement observed up to $65 \mathrm{GHz}$ confirms the validity of the series resistance extraction approach.

\section{CONCLUSION}

Error terms in the extraction of series resistances for HBT devices using known methods based on measured Sparameters have been identified. It has been shown that the 


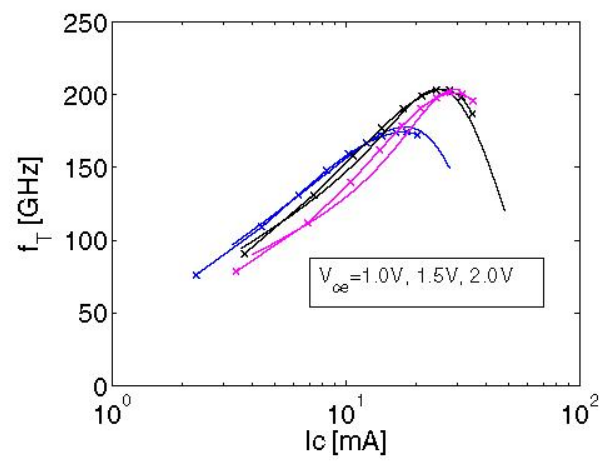

Fig. 7. Bias dependence of cutoff frequency. Symbols: measurements, solid line: model response

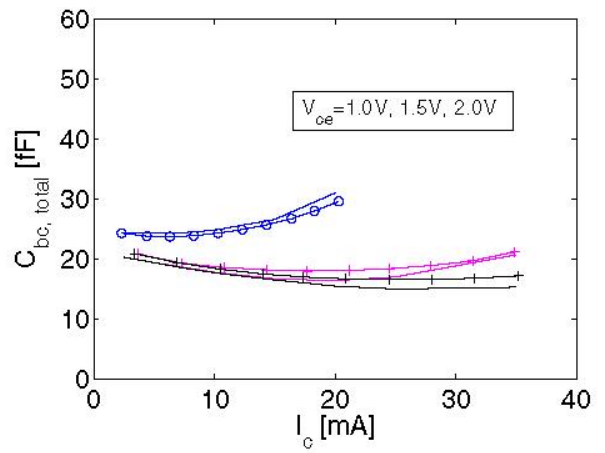

Fig. 8. Bias dependence of total base-collector capacitance. Symbols: measurements, solid line: model response

access emitter resistance found from S-parameters in the saturation region is overestimated due to the presence of an intrinsic collector resistance. The access emitter resistance extracted from the forward active region allows the intrinsic collector resistance to be extracted. The determined series resistances were applied to the large-signal model extraction of an integrated InP DHBT device. It was found that the extracted intrinsic collector resistance lead to accurate modeling of the static $I_{c}-V_{c e}$ characteristics in the saturation region. The excellent fitting of the cut-off frequency and base-collector capacitance versus collector current and S-parameters versus frequency confirms the validity of the approach.

\section{ACKNOWLEDGMENT}

The authors would like to thank the European Commission for support under the 6th framework programme to the project 'Opto-electronic integration for 100 Gigabit Ethernet Optical Networks (GIBON)'.

\section{REFERENCES}

[1] L. H. Camnitz et al., "An Accurate Large Signal High Frequency Model for GaAs HBTs", 1996 GaAs IC Symposion, pp. 303-306, 1996.

[2] H.-C Wu et al., "Extraction of collector resistances for device characterization and compact models", Solid-State Electronics , pp. 1475-1478, 2006.

[3] L. Jun et al., "A New Direct Method for HBT B-E \& B-C Distributed Capacitances and Intrinsic Resistances Extraction", 2005 Proc. APMC, 2005.

[4] Y. Gobert et al., "A Physical, Yet Simple Small-Signal Equivalent Circuit for the Heterojunction Bipolar Transistor", IEEE Trans. Microwave Teory and Tech., Vol. 45, No. 1, pp. 149-153, Jan. 1997.

[5] M. W. Dvorak et al., "On the Accuracy of Direct Extraction of the Heterojunction-Bipolar-Transistor Equivalent-Circuit Model Parameters $C_{\pi}, C_{b c}$, and $R_{e}$ ", IEEE Trans. Microwave Teory and Tech., Vol. 51, No. 6, pp. 1640-1649, Jun. 2003.

[6] Y. Suh et al., "Direct Extraction Method for Internal Equivalent Circuit Parameters of HBT Small-Signal Hybrid-П Model", 2000 EEE MTT-S, pp. 1401-1404, 2000.

[7] W. J. Kloosterman et al., "Improved Extraction of Base and Emitter Resistance from Small-Signal High Frequency Admittance Measurements", 1999 IEEE BCTM, pp. 93-96, 1999.

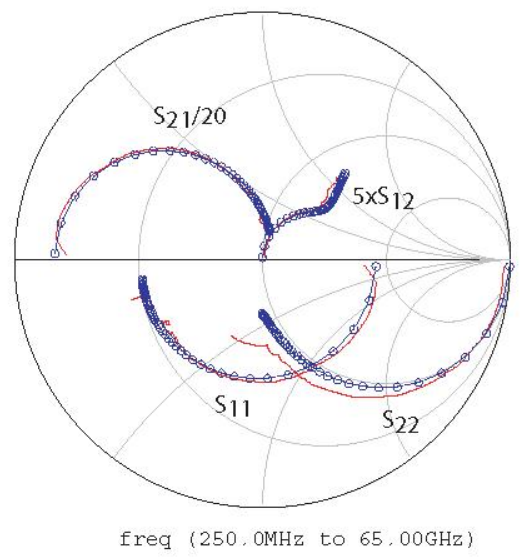

Fig. 9. S-parameters versus frequency $\left(I_{c}=24.5 \mathrm{~mA}, V_{c e}=1.5 \mathrm{~V}\right)$. Symbols: model response, solid line: measurements. 